\title{
Harmonic generation for atoms in fields of varying ellipticity: Single-active-electron model with Hartree-Fock potential
}

\author{
I. A. Ivanov* and A. S. Kheifets \\ Research School of Physical Sciences and Engineering, The Australian National University, \\ Canberra, Australian Capital Territory 0200, Australia
}

(Received 19 March 2009; published 15 May 2009)

\begin{abstract}
We propose a theoretical procedure for calculating harmonics yield from atoms based on the solution of a three-dimensional time-dependent Schrödinger equation. The atomic system is described by means of the Hartree-Fock potential, including the exchange interaction. We present results for the harmonics generation in lithium and rubidium in situation when ellipticity of the driving laser changes with time. These results show that harmonics are formed primarily at the intervals of time when the driving laser field has nearly zero ellipticity. This can be used for generation of short (hundreds of attoseconds) bursts of radiation.
\end{abstract}

DOI: 10.1103/PhysRevA.79.053827

PACS number(s): 42.65.Ky, 32.80.Fb

\section{INTRODUCTION}

Atom placed in a sufficiently strong pulse of electromagnetic (EM) field emits radiation predominantly at the odd multiples of the carrier frequency of the pulse. This is the well-known process of the high-harmonic generation (HHG). Remarkably, many features of the HHG process can be understood on the basis of a simple three-step model [1]. At the first step, at some moment of time $t_{0}$, the atomic electron undergoes a tunneling ionization which leaves it predominantly in the continuum with zero velocity. For some values of $t_{0}$, the classical trajectory of the electron in the EM field will eventually return to the nucleus where the electron can recombine and emit a photon. This essentially classical model explains many characteristic features of the HHG process such as existence of the plateau in the spectrum [2,3].

The reason of the success of the classical approach in describing this process becomes clear in the framework of the quantum-mechanical description [4]. Instead of a classical description of electron trajectories in the EM field, this approach relies on the quantum-mechanical description of the motion of the released electron. Such description is possible in the framework of the so-called strong-field approximation (SFA), which neglects the influence of the atomic potential on the motion of the released electron during the second stage of the HHG process. For quantum-mechanical description of the electronic motion in presence of the EM field only, the well-known Volkov states can then be employed, and the problem can be treated analytically.

Calculation of the amplitudes in this approach can be simplified by the use of the saddle-point method (which is justified by the by the conditions of the validity of the SFA). Thus, classical recurrent trajectories naturally emerge as extrema in the saddle-point analysis of the quantummechanical amplitudes computed within the SFA.

Thus, both classical and quantum analysis emphasize importance of the existence of the electron trajectories revisiting the nucleus after the ionization event. Simple classical considerations give two such trajectories per cycle for the harmonics at the cutoff region, and four harmonics (two pairs

\footnotetext{
*Corresponding author; igor.ivanov@.anu.edu.au
}

of the so-called "long" and "short" trajectories) for the plateau region. Each of these trajectories produce a short (attosecond) burst of radiation [5]. Interference of these attosecond bursts produce the observed harmonic spectrum [6].

This picture of the harmonics formation based on the existence of the electron trajectories revisiting the nucleus is both physically transparent and fruitful. It is clear that if we modify electron trajectories so that the recollision event at the origin no longer takes place, we may dramatically modify the harmonics emission. This happens, for example, if elliptically polarized light is used as driving pulse. No revisiting trajectories exist for such field configuration; consequently, harmonics formation should be greatly suppressed. That this is indeed the case was shown in works $[7,8]$. If ellipticity of the driving pulse is varying in time, then harmonics will be produced predominantly at the intervals of time when polarization is close to linear. This opens up a possibility of producing very short (attosecond) pulses of light, which can be used to literally track motion of electrons in atoms and molecules [9].

One such scheme, proposed in [10], used two pulses with different carrier frequencies. Another scheme, the so-called polarization gate technique, proposed in [11,12], employed a field configuration consisting of two pulses of left and right circular polarizations separated by a time delay. For such configuration polarization of the total EM field is close to linear only for the time interval on which both pulses significantly overlap. Simulations performed in works $[11,12]$ showed that this field configuration could be used to produce single attosecond pulses.

The tool employed for theoretical simulations in these works was the SFA which, as we mentioned above, neglected effects of the atomic potential on the motion of the ionized electron. This is a very good approximation for the highorder harmonics in the tunneling regime (small values of the Keldysh parameter $\gamma=\sqrt{I_{p} / 2 U_{p}}$, where $I_{p}$ is the ionization potential and $U_{p}$ is the ponderomotive potential). For the low-order harmonics, or for the regime, characterized by values of the Keldysh parameter close to 1 (the regime transitional between the multiphoton and tunneling), the effect of the atomic potential may dramatically modify the harmonics spectrum. In particular, various possible multiphoton resonances may change harmonics intensities by several orders of magnitude [13-15]. 
The aim of this paper is to study the harmonics formation in this regime by means of the theoretical approach, which takes into account the atomic structure. Below we solve numerically the full three-dimensional (3D) time-dependent Schrödinger equation (TDSE) for two atomic systems-the $\mathrm{Rb}$ and $\mathrm{Li}$ atoms in presence of the driving pulse of varying ellipticity-and study formation of the harmonics in this case. Details of the theoretical approach we use and analysis of the results are given below.

\section{THEORY}

We rely on the single active electron approximation (SAE) to describe the target atom. We describe the field-free atom in the ground state by solving a set of self-consistent Hartree-Fock equations [16]. One-electron excitations from the valence shell are treated within the frozen-core HartreeFock (FCHF) approximation. The field-free atomic Hamiltonian in our model is, thus, a nonlocal integrodifferential operator. To account for the polarization of the core, the polarization potential $V(r)=-\frac{\alpha}{2\left(r^{2}+r_{0}^{2}\right)^{2}}$ is added to the Hamiltonian [17]. Here $\alpha$ is atomic polarizability, the parameter $r_{0}$ is chosen such that the energy of the first-excited atomic state is reproduced accurately.

We are solving the TDSE for the atomic system in the external EM field in the weak form,

$$
\left\langle\Phi \mid\left(i \frac{\partial}{\partial t}-\hat{H}_{\text {atom }}-\hat{H}_{\text {int }}(t)\right) \Psi\right\rangle=0,
$$

where $|\Phi\rangle$ is any vector from the subspace $\mathcal{V}$ of the Hilbert space $\mathcal{H}$ which is defined as follows.

We define the set of the so-called pseudostates $[17,18]$ diagonalizing the nonlocal field-free atomic Hamiltonian in a suitable square-integrable basis [18],

$$
\left\langle f_{n l}^{N}\left|\hat{H}_{\text {atom }}\right| f_{n^{\prime} l^{\prime}}^{N}\right\rangle=E_{n l} \delta_{n n^{\prime}} \delta_{l l^{\prime}} .
$$

Use of the set of pseudostates to build $\mathcal{V}$ is motivated by the fact that for a sufficiently large basis size $N$, the pseudostates span a large portion of the Hilbert space. Weak solution (1) can, therefore, be expected to approximate accurately the exact solution of the TDSE. To build a basis set in Eq. (2), we use a set of the Laguerre functions.

For atomic systems with a nonempty core, the pseudostates with lowest energies in Eq. (2) may correspond to the core states of the field-free FCHF atomic Hamiltonian. These pseudostates must be excluded from consideration.

The subspace $\mathcal{V}$ in which the solution of Eq. (1) is sought is, thus, a span of all pseudostates defined by Eq. (2) which do not belong to the atomic core,

$$
\Psi(\boldsymbol{r}, t)=\sum_{n l m \notin \text { core }} a_{n l m}(t) f_{n l}(\boldsymbol{r}) Y_{l m}(\hat{\boldsymbol{r}}) .
$$

To describe interaction of the atom and the external electromagnetic field, we use the length gauge. In the present setup, when the atom interacts with two pulses of right and left circular polarizations arriving with the time delay $\Delta$, the interaction Hamiltonian can be written as

$$
\hat{H}_{\text {int }}(t)=E_{0}\left[x f_{1}(t) \cos \omega t+y f_{2}(t) \sin \omega t\right],
$$

where $f_{1}(t)=f(t)+f(t-\Delta), f_{2}(t)=f(t)-f(t-\Delta)$, and $f(t)$ is the function describing envelope of the pulse. The envelope function $f(\tau)$ is constant for $\tau \in(T, 4 T)$, where $T=2 \pi / \omega$ is a cycle duration corresponding to the carrier frequency in $\mathrm{Eq}$. (4). The fields are ramped on and off smoothly (so that field strength and its first time-derivative remain continuous) over one cycle duration. The total duration of the left- and rightpolarized pulses of EM radiation is, therefore, $T_{1}=6 T$. The atom, thus, interacts with the combined pulse during the interval of time $\left(0, T_{2}\right)$, where $T_{2}=6 T+\Delta$. In this interval the left- and right-polarized pulses overlap within the time interval $(\Delta, 6 T)$. On the interval $(\Delta+T, 5 T)$ of duration $4 T-\Delta$ both overlapping pulses are completely ramped on; the resulting EM field is, therefore, polarized linearly along the $x$ axis.

The last ingredient of our model is the absorbing potential. Pseudostates (2) are computed within a box of the finite size (200 a.u. in the present calculation). We have to exclude the possibility of the reflections of the wave packets from the boundaries of the box, which otherwise may lead to the appearance of the spurious harmonics in the spectrum. This can be done either by means of a mask function or absorbing potential. We use the absorbing potential $-i W(\boldsymbol{r})$ which is chosen as a smooth function, zero for $r \leq 180$ a.u., and continuously growing to a constant $-i W_{0}$ with $W_{0}=2$ a.u. outside this region.

With, thus, defined the total Hamiltonian, Eqs. (1) and (3) lead to a system of differential equations for the coefficients $a_{j}(t)$. The solution of this system is found by means of the Crank-Nicholson method. The spectrum of the harmonics is calculated as [2]

$$
|d(\omega)|^{2}=\left|\int_{0}^{T_{2}} e^{-i \omega t} d_{x}(t) d t\right|^{2} .
$$

Here $d(t)=\langle\Psi(t)|x| \Psi(t)\rangle$ is expectation value of the dipole momentum and $T_{2}=6 T+\Delta$ is total duration of the combined pulse.

In the calculations we present below, the pseudostates with angular momenta $l<l_{\max }=10$ were included in Eq. (3). The only reason for this limitation is computational feasibility of the problem. The TDSE we have to solve is a fully three-dimensional problem which implies that in Eq. (3) the terms with all possible projections of the momenta are to be retained. Experience shows that a good convergence with respect to the basis size can be achieved for $N \approx 40$ for each value of $l$ in Eq. (2). The overall size of the problem grows, therefore, as $N l_{\max }^{2}$, which compels us to choose a relatively low value for $l_{\max }$. The limitation which such choice of $l_{\max }$ imposes is that we cannot consider formation of the harmonics of really high orders. For the modestly strong electric fields of the order of 0.005 a.u., which we consider below, our choice of $l_{\max }$ enables us to study formation of harmonics of the order not exceeding $l_{\max }$.

This restriction could be weakened. It was shown $[19,20]$ that if velocity gauge is used to describe the atom-EM field interaction, a considerably smaller number of the angular- 

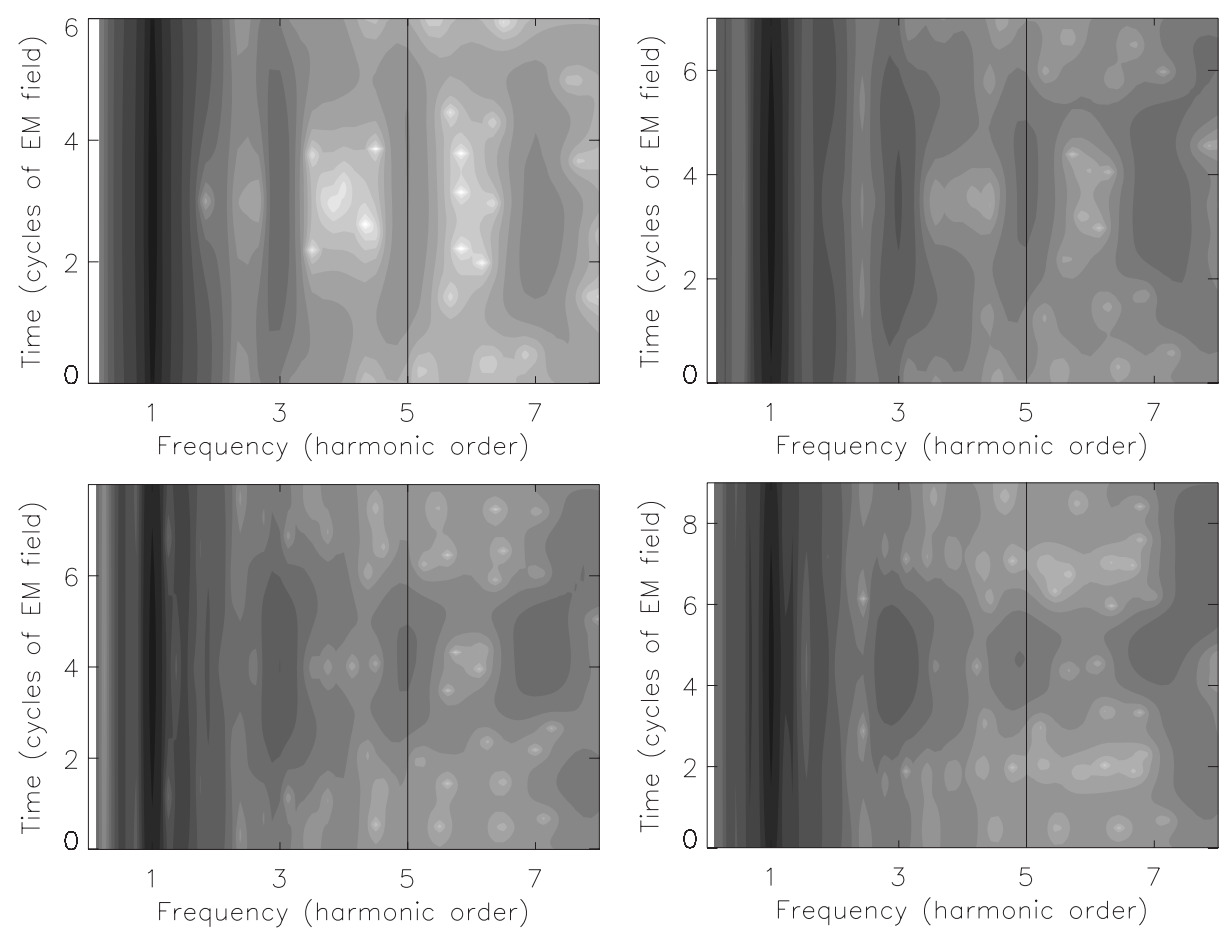

FIG. 1. Wavelet timefrequency spectrum of $\mathrm{Rb}$ for $\Delta$ $=0, T, 2 T, 3 T ; x_{0}=20$. The darker shades of gray represent more intense signals. momentum states in the expansion Eq. (3) is required to represent solution of the TDSE accurately. This circumstance may offer an important advantage if for the problem considered accurate solution of TDSE in the length gauge requires a large number of the angular-momentum states to be retained in the Eq. (3). Two examples of such problems are calculation of above threshold ionization (ATI) peaks of high orders [19], or description of the formation of high-order harmonics.

As we mentioned in Sec. I, the goal of the present work is to study formation of the harmonics of the relatively low orders. For this process, the gain in the speed of the convergence of expansion (3), which use of the velocity gauge offers, is not so important, and length gauge can be used efficiently.

Even for relatively low-order harmonics considered below, we shall see the essential features of the HHG process, which can be interpreted as the fingerprints of the electron trajectories. We shall also see how these features are affected by varying the time delay between the right- and leftpolarized pulses of the EM radiation.

\section{RESULTS}

We consider below the $\mathrm{Rb}$ and $\mathrm{Li}$ atoms subjected to the EM radiation with the following parameters entering Eq. (4). For the $\mathrm{Rb}$ atom we use Eq. (4) $E_{0}=0.0022$ a.u., $\omega$ $=0.2 \mathrm{eV}$. For the $\mathrm{Li}$ atom we use $E_{0}=0.0035$ a.u. and $\omega$ $=0.16 \mathrm{eV}$. For this choice of the field parameters we have (for the time interval where combined field is nearly polarized, which, as we shall see, the interval which matters for the generation of harmonics) the values of the Keldysh parameter 0.5 for $\mathrm{Li}$ and 0.95 for $\mathrm{Rb}$. The particular choice of the field parameters is arbitrary to a degree, as long as we stay in the soft tunneling regime, characterized by the values of the Keldysh parameter not much less than 1 . This is the regime we wished to study by means of the described above TDSE-based approach (as we mentioned in Sec. I, deeper in the tunneling regime, the effect of the atomic potential becomes less important, and computationally less demanding SFA-based methods of calculation could be used).

We perform our analysis of the HHG process by applying the wavelet transform of the dipole operator $d(t)$ in Eq. (5). The wavelet transform of a square-integrable function $d(t)$ is defined as [21]

$$
T_{\Psi}(\omega, \tau)=\int d(t) \sqrt{|\omega|} \Psi^{*}(\omega t-\omega \tau) d t
$$

This transform is generated by a function $\Psi(x)$ (the mother wavelet). As a mother wavelet we use the Morlet wavelet $\Psi(x)=x_{0}^{-1} \exp (-i x) \exp \left[-x^{2} /\left(2 x_{0}^{2}\right)\right]$.

The wavelet transform [22-24], or closely related techniques such as the Gabor transform [25], allow us to track the process of harmonics formation in time combining both the frequency and temporal resolution of a signal. For the Morlet wavelet employed in the present work, the balance between resolutions in the frequency and temporal domains is defined by the parameter $x_{0}$. As can be seen from the expression for the Morlet wavelet, the resolution in the temporal domain is independent of the frequency and increases with decrease in the parameter $x_{0}$.

Figures 1 and 2 show decrease in the time interval during which harmonics are formed with increasing time delay $\Delta$. With increasing $\Delta$ harmonics formation is confined to ever decreasing time interval in which the light polarization is linear.

To see in more details how the harmonics are formed in time, we take a vertical cut of the 3D plots in Figs. 1 and 2 along the fixed frequency corresponding to the fifth har- 

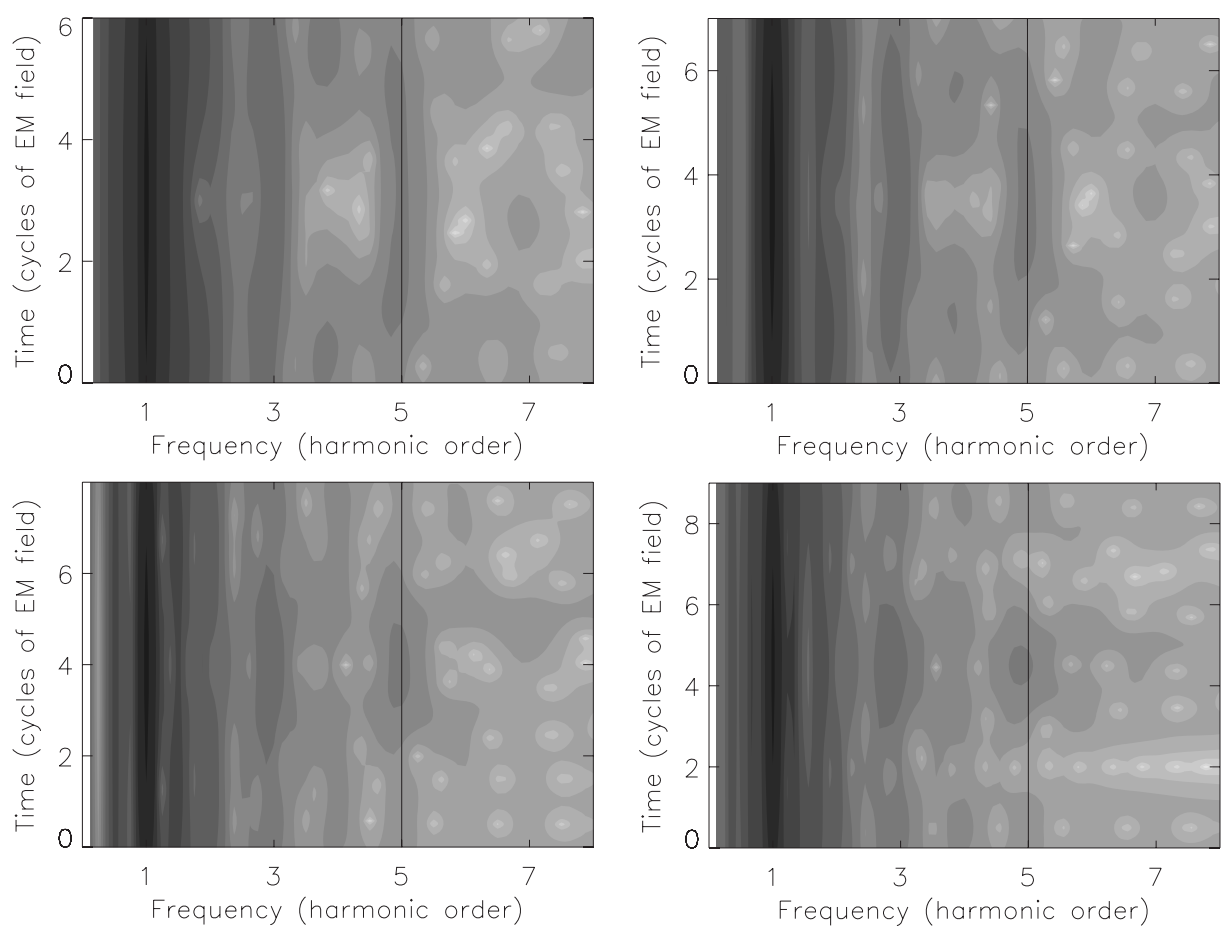

FIG. 2. Wavelet timefrequency spectrum of Li for $\Delta$ $=0, T, 2 T, 3 T ; x_{0}=20$. monic (as indicated on the Figs. 1 and 2). In Figs. 3 and 4 we show the time-frequency spectra for the $\mathrm{Li}$ and $\mathrm{Rb}$ atoms for the fixed frequencies corresponding to the fifth harmonic. To achieve better resolution in the temporal domain, the wavelet parameter $x_{0}$ for these spectra has smaller value $x_{0}=5$.

The spectra exhibit the feature which we already noted above - the gradual shortening of the interval on which harmonics are formed with increase in $\Delta$. The feature which becomes visible under better temporal resolution of Figs. 3 and 4 is the presence of local maxima (two maxima per cycle of the driving pulse) for the amplitude of the wavelet transform on the interval of time on which polarization of the combined pulse is close to linear.

It is known [23] that in the case of a linearly polarized driving pulse, the amplitude of the wavelet transform for the lowest-order harmonics is a smooth slowly varying function of time. This follows from the fact that the lowest-order harmonics are produced predominantly by the multiphoton ionization mechanism [23]. In this regime, the probability of absorbing $N$ photons is proportional to the $N$ th power of the intensity of the EM field.

On the other hand, for the purely tunneling regime (which is at work for the formation of the harmonics at the plateau region and near the cutoff) we would have four maxima per cycle (harmonics at the plateau) or two maxima per cycle (near cutoff harmonics), corresponding to the number of the available classical trajectories revisiting the nucleus.

Superficially, the timing spectra in Figs. 3 and 4 are, therefore, reminiscent of the wavelet time spectra for the near cutoff harmonics, where two trajectories per cycle contribute to the formation of a harmonic. The harmonics we consider here are, of course, far from the cutoff. Presence of the temporal structures in Figs. 3 and 4 signifies the fact that for this harmonic the mechanism of its formation is transitional between the multiphoton and tunneling mechanisms. The traces of the trajectories are already present, manifesting themselves as two maxima of the amplitude of the wavelet transform, These maxima, however, are not yet resolved in the components corresponding to the short and long trajectories of the classical model which appear for the higherorder harmonics at the plateau [24].

The presence of these four maxima for the plateau harmonics enables to produce up to four short (attosecond) pulses per cycle of the driving field. For the field configuration considered in Ref. [12], where the total EM field was linearly polarized for the duration of approximately one
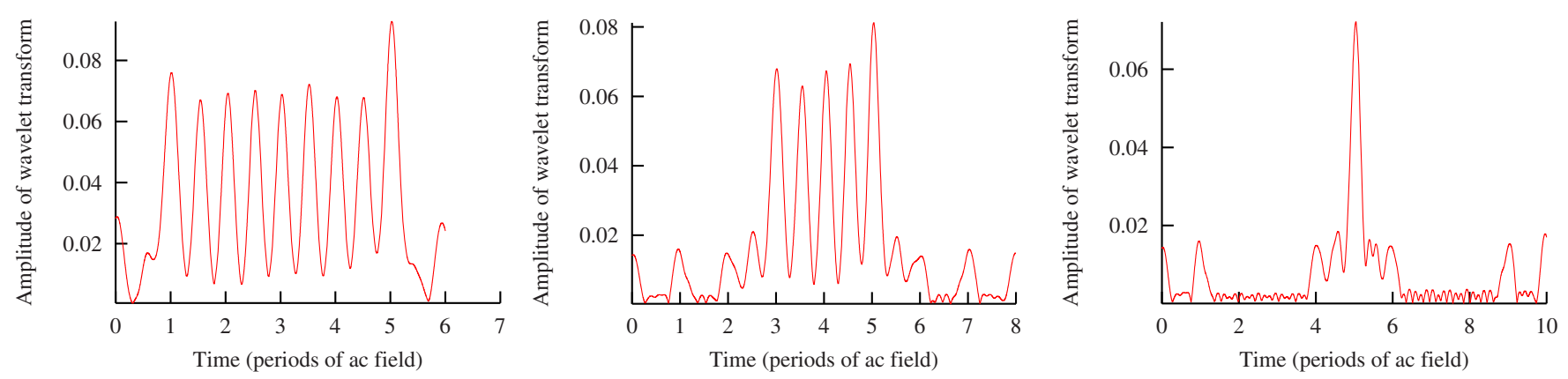

FIG. 3. (Color online) Wavelet time spectrum of Rb for the fifth harmonic for (from left to right) $\Delta=0,2 T, 4 T ; x_{0}=5$. 

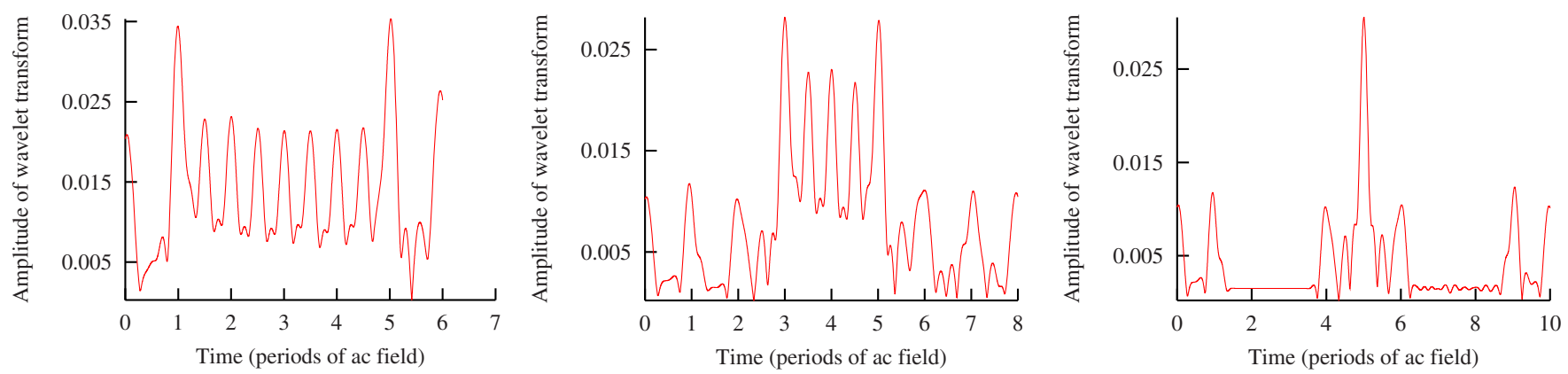

FIG. 4. (Color online) Wavelet time spectrum of Li for the fifth harmonic for (from left to right) $\Delta=0,2 T, 4 T ; x_{0}=5$.

cycle, the simulation based on the SFA approximation predicted appearance of several attosecond pulses.

In our case, the situation is different in one respect. The wavelet transform indicates that we can expect to produce two bursts of radiation per cycle of the driving field. That this in indeed so can be seen in Figs. 5 and 6 where we present the results of the windowed Fourier transform. To produce these data, we apply the inverse Fourier transform using the Fourier coefficients defined in Eq. (5) weighted with a window function. For such a function we use a Gaussian centered at the frequency corresponding to the fifth harmonic with the width of $2 \omega$. The exact form of the window function is not so important for qualitative conclusions. In Ref. [12], for example, the rectangular window was used.

Thus obtained data for the intensity of the signal tell essentially the same story as the wavelet transform results we discussed above. The two local maxima per cycle of the driving pulse result in a two short pulses of radiation produced every cycle. With increasing time delay $\Delta$, the timing when these pulses are emitted is restricted to ever shortening interval of time in which the driving pulse polarization is close to linear.

\section{CONCLUSION}

We applied a computational procedure which relies on the Hartree-Fock potential, including the nonlocal exchange interaction to solve the TDSE for the $\mathrm{Li}$ and $\mathrm{Rb}$ atoms interacting with two pulses of right and left circular polarizations arriving with the time delay $\Delta$. Numerical solution of TDSE was used to study the process of the harmonics generation for the regime intermediate between tunneling and multiphoton ionization. The use of such a field configuration was proposed earlier in Refs. $[11,12]$ as a means to produce attosecond pulses of radiation. Theoretical treatment in these works relied on the SFA which neglected all effects of the atomic core. Use of this approximation to describe harmonics formation is legitimate for high-order harmonics at the plateau or near the cutoff.

In the present work, we consider low-order harmonics which, as the wavelet analysis suggests, are intermediate between the multiphoton and tunneling regimes. The effect of the atomic structure can be important for these harmonics. Even though some details of the harmonics generation are different in this regime, (such as the number of pulses pro-
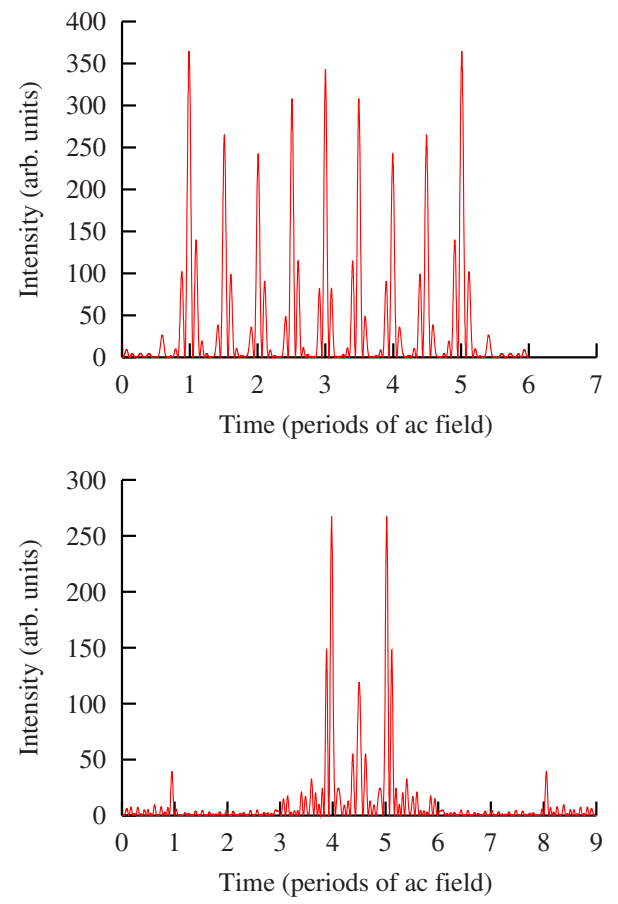

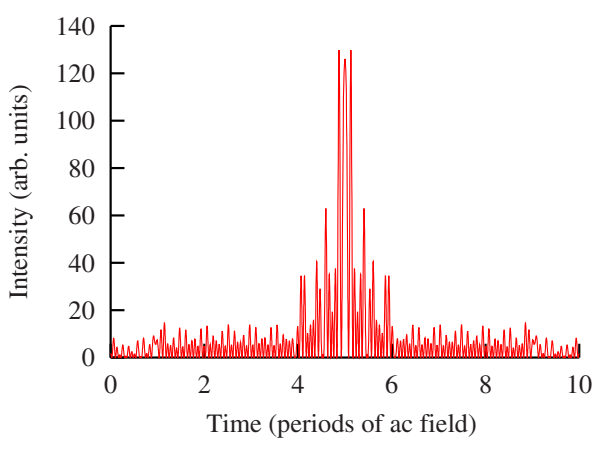

FIG. 5. (Color online) Intensity in the spectral window with the width $2 \omega$ near the fifth harmonic for $\mathrm{Rb}$ for $\Delta$ $=0,2 T, 3 T, 4 T$. 

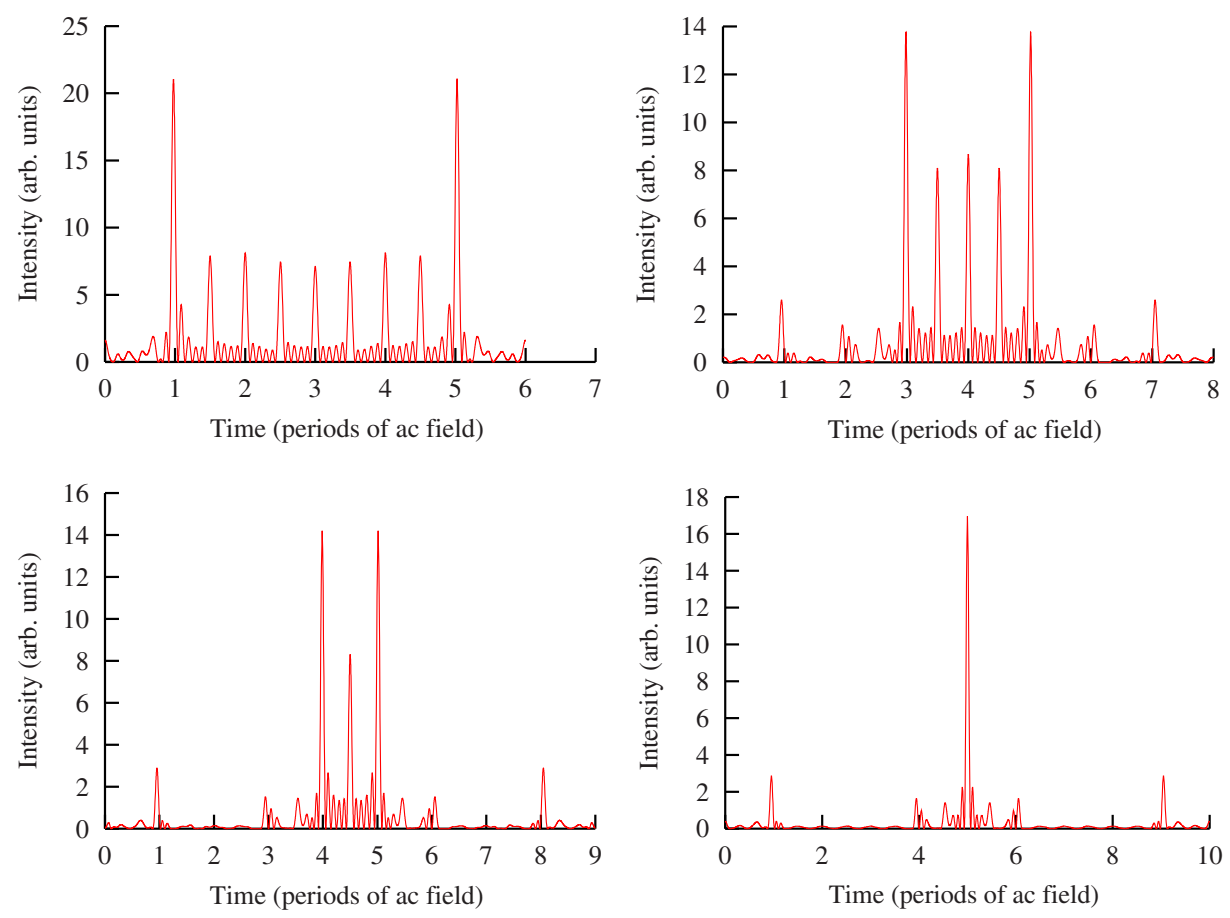

FIG. 6. (Color online) Intensity in the spectral window with the width $2 \omega$ near the fifth harmonic for $\mathrm{Li}$ for $\Delta=0,2 T, 3 T, 4 T$. duced per cycle of the driving field as compared with the case of the plateau harmonics), this regime can also be used for generation of short pulses. All results were illustrated for the fifth harmonic. Analogous conclusions hold for the higher harmonics $\mathrm{H} 7$ and $\mathrm{H} 9$, which we were able to consider in the present numerical calculation. For higher-order harmonics, as we noted, the treatment based on the numerical solution of TDSE becomes problematic due to a very rapid increase in the basis size. For higher-order harmonics, however, we enter the region of validity of the SFA. This approximation was employed in Ref. [12] to consider har- monics formation for the present field configuration. These two approaches, therefore, complement each other covering all the harmonics spectra.

\section{ACKNOWLEDGMENTS}

The authors acknowledge support of the Australian Research Council in the form of the Discovery Grant No. DP0771312. Resources of the National Computational Infrastructure (NCI) Facility were employed.
[1] P. B. Corkum, Phys. Rev. Lett. 71, 1994 (1993).

[2] J. L. Krause, K. J. Schafer, and K. C. Kulander, Phys. Rev. A 45, 4998 (1992).

[3] A. L'Huillier and Ph. Balcou, Phys. Rev. Lett. 70, 774 (1993).

[4] M. Lewenstein, P. Balcou, M. Y. Ivanov, A. L'Huillier, and P. B. Corkum, Phys. Rev. A 49, 2117 (1994).

[5] P. Antoine, A. L'Huillier, and M. Lewenstein, Phys. Rev. Lett. 77, 1234 (1996).

[6] E. Priori et al., Phys. Rev. A 61, 063801 (2000).

[7] P. Antoine, A. LHuillier, M. Lewenstein, P. Salières, and B. Carré, Phys. Rev. A 53, 1725 (1996).

[8] B. Shan, S. Ghimire, and Z. Chang, Phys. Rev. A 69, 021404(R) (2004)

[9] A. Scrinzi, M. Yu. Ivanov, R. Kienberger, and D. M. Villeneuve, J. Phys. B 39, R1 (2006).

[10] M. Ivanov, P. B. Corkum, T. Zuo, and A. Bandrauk, Phys. Rev. Lett. 74, 2933 (1995).

[11] V. T. Platonenko and V. V. Strelkov, J. Opt. Soc. Am. B 16, 435 (1999).

[12] Z. Chang, Phys. Rev. A 70, 043802 (2004).

[13] R. A. Ganeev, J. Phys. B 40, R213 (2007).
[14] D. B. Milošević, J. Phys. B 40, 3367 (2007).

[15] I. A. Ivanov and A. S. Kheifets, Phys. Rev. A 78, 053406 (2008).

[16] L. V. Chernysheva, N. A. Cherepkov, and V. Radojevic, Comput. Phys. Commun. 11, 57 (1976).

[17] I. Bray and A. T. Stelbovics, Adv. At., Mol., Opt. Phys. 35 209 (1995)

[18] I. Bray, Phys. Rev. A 49, 1066 (1994).

[19] E. Cormier and P. Lambropoulos, J. Phys. B 29, 1667 (1996).

[20] M. Nurhuda and F. H. M. Faisal, Phys. Rev. A 60, 3125 (1999).

[21] Y. Y. Tang, L. H. Yang, J. Liu, and H. Ma, Wavelet Theory and Its Application to Pattern Recognition (World Scientific, Singapore, 2000).

[22] X. Chu and Shih-I Chu, Phys. Rev. A 64, 021403(R) (2001).

[23] X. M. Tong and Shih-I Chu, Phys. Rev. A 61, 021802(R) (2000).

[24] X. M. Tong and S. I. Chu, J. Phys. B 32, 5593 (1999).

[25] R. Taïeb, V. Véniard, J. Wassaf, and A. Maquet, Phys. Rev. A 68, 033403 (2003). 